\title{
Generalization and application of the Cauchy-Poisson method to elastodynamics of a layer and the Timoshenko equation
}

\author{
Selezov I. \\ Institute of Hydromechanics, NASU, \\ 8/4 Zheliabov Str., 03680 Kyiv, Ukraine
}

(Received 21 March 2018)

\begin{abstract}
The Cauchy-Poisson method is extended to $n$-dimensional Euclidean space so that to obtain partial differential equations (PDEs) of a higher order. The application in the construction of hyperbolic approximations is presented, generalizing and supplementing the previous investigations. Restrictions on derivatives in Euclidean space are introduced. The hyperbolic degeneracy by parameters and its realization in the form of necessary and sufficient conditions are considered. As a particular case of 4-dimensional Euclidean space, keeping operators up to the 6th order, we obtain a generalized hyperbolic equation of transverse (bending) vibrations of plates with coefficients depending only on the Poisson number. Numerical calculations are carried out and presented. This equation includes, as special cases, all the known equations of Bernoulli-Euler, Kirchhoff, Rayleigh, Timoshenko. It should be noted that the refined equation of bending oscillations of a beam, firstly presented by Timoshenko, must be considered as the development of Maxwell's and Einstein's investigations on the perturbation propagation with finite velocity in media. For the first time, the conformity with the Cosserat theory is noted.
\end{abstract}

Keywords: Cauchy-Poisson method, Euclidean space, partial differential equation (PDE), elastodynamics, layer, hyperbolic approximations, Timoshenko equation.

2000 MSC: $74 \mathrm{H} 10,74 \mathrm{~J} 05,74 \mathrm{~K} 20$

UDC: 531.4

DOI: $10.23939 / \mathrm{mmc} 2018.01 .088$

\section{Introduction}

Modeling wave processes in real systems leads to the need to investigate degenerate operators. This problem has always been relevant in mathematics. Great interest is the modeling wave propagation in degenerate hyperbolic systems so that the finite velocity of perturbation propagation is satisfied.

The construction of hyperbolic equations describing real phenomena of perturbation propagation with finite velocity is an important problem. Such modeling is based on Maxwell's research (Maxwell, 1864) [1]. After modeling electromagnetic field, using a deep analysis Maxwell showed the finite velocity of perturbation propagation in the gas, in contrast to the traditional Boltzmann model (Maxwell, 1867) [2]. The construction of hyperbolic models as the development of Maxwell's investigations was realized by Einstein (Einstein, 1950) [3] and in the study of gravitational waves developed by Weber (1950) [4]. Over the years of research and at last experimental detection of the speed of propagation of gravitational waves, the Nobel Prize has been awarded to researchers in 2017: B. C. Barish, K. S. Thorne, R. Weiss.

After Maxwell, generalized hyperbolic models describing the propagation of heat, diffusion, and others have been developed, cited in (Selezov and Krivonos, 2015) [5]. We also note the latest study on the injection of a medical preparation into tissue, where a new generalization of the diffusion equation to a hyperbolic equation is presented (Selezov \& Kryvonos, 2017) [6]. From the mathematical point of view, in essence, a second-order parabolic operator (predicting the perturbation propagation with infinite velocity) was generalized to a hyperbolic operator of the second order (but now describes the perturbation propagation with finite velocity). The method of solving the problem of elastodynamics 
for a layer by expansion in a small thickness coordinate was proposed in 1828-29 in works (Cauchy, 1828) [7] and (Poisson, 1829) [8]. This reduces the dimensionality of the problem by one, i.e. reduces the three-dimensional problem to a two-dimensional one which was essentially used in the construction of the theory of plates and shells. The method was generalized to n-dimensional Euclidean space in 2000 by Selezov (Selezov, 2000) [9] and without assumptions was considered in (Selezov and Kryvonos, 2015) [5]. The study presented earlier in the $n$-dimensional Euclidean space requires the introduction of additional restrictions on degeneracy with respect to small parameters.

In this paper we present a generalization of the Cauchy-Poisson method to $n$-dimensional Euclidean space and introduce a restriction on the derivatives. We consider hyperbolic degeneracy and the construction of hyperbolic approximations of higher order. In the particular case $n=4$, the wave hyperbolic equations for the elastic layer are obtained. This approximation is of a higher order, in contrast to known studies. It is especially first noted that Timoshenko's equation for flexural oscillations of a beam is a non-trivial generalization of the classical theory.

\section{Generalization of the Cauchy-Poisson method on $n$-dimensional Euclidean space $\mathbb{R}^{n}$}

In Euclidean space $\mathbb{R}^{n}$ with coordinates $x^{q}, q=\overline{1, n}$, we consider a mathematical model represented by a finite system of PDEs, for which a boundary value problem is posed in a domain $\Omega \times\left[0, X^{m}\right]$, $X^{m}>0$ bounded by hypersurfaces $x^{s}= \pm h^{s}, h^{s}>0$ (the index $s$ is fixed):

$$
\Omega=\left\{x \in \mathbb{R}^{n}:-\infty<\left(x^{1}, x^{2}, \ldots, x^{s-1}, x^{s+1}, \ldots, x^{n-1}\right)<\infty, x^{n} \geqslant 0,-h^{s} \leqslant x^{s} \leqslant h^{s}\right\} .
$$

We assume that the hypersurfaces $x^{s}= \pm h^{s}$ are removed from the middle hypersurface $x^{s}=0$ and that the expansion is relative to $x^{s}=0$. We consider the case when conditions are given on hypersurfaces $x^{s}= \pm h^{s}$. It is assumed that the model depends on a finite number $\nu$ of parameters $\varepsilon_{r}, r=\overline{1, \nu}$. Formally, such a model can be defined as a system $k$ of PDEs of the $p$-th order with $k$ unknowns $u_{i},(i=\overline{1, k})$ and $n$ arguments (Dunford \& Schwartz, 1969) [10]

$$
F_{i}(x^{1}, \ldots, x^{n} ; u_{1}, \ldots, u_{k} ; u_{1,1}, \ldots, u_{k, n} ; \ldots, u_{1}, \underbrace{1 \ldots 1}_{P \text { times }}, \ldots, u_{k, \underbrace{n \ldots n}_{P \text { times }}}^{n} ; \varepsilon_{1}, \ldots, \varepsilon_{\nu})=P_{i}\left(x^{1}, \ldots, x^{n}\right) \in \Omega .
$$

The following system of boundary conditions on hypersurfaces $x^{s}=-h^{s}, x^{s}=h^{s}$ is given

$$
\left.f_{j}(x^{1}, \ldots, x^{n} ; u_{1}, \ldots, u_{k} ; u_{1,1}, \ldots, u_{k}, \underbrace{n \ldots n}_{(P-1) \text { times }} ; \varepsilon_{1}, \ldots, \varepsilon_{\nu})\right|_{x^{s}= \pm h^{s}}=Q_{j}^{ \pm}, \quad j=\overline{1,(k \cdot p)} .
$$

Here the index after the "comma" denotes differentiation with respect to the corresponding coordinate. In general case $p \neq n$. The function $F_{l}$ depends on all possible partial derivatives of the $p$-th order inclusive, the position of the hypersurface can depend on $u_{i}$ and their derivatives. The solution of the boundary value problem (1), (2) consists in the definition of functions $u_{i}$, which transform equations (1) into identities, and in the choice of the set of these functions such that they satisfy conditions (2).

The assumption of smallness of parameters. The parameters $\varepsilon_{\nu}$ are assumed to be small, $\varepsilon_{\nu} \ll 1$. As a result, degeneration by parameters $\varepsilon_{1}, \ldots, \varepsilon_{\nu}$ can be considered. In the case of parameter degeneracy, the problem can be essentially simplified: a reduction in the order of the system of PDEs, partial decomposition, etc. This problem can be considered as a mapping of a partial differential operator from $\mathbb{R}^{n}$ in $\mathbb{R}^{n-1}$.

Deviations of hypersurfaces $x^{s}= \pm h^{s}$ from a hypersurface $x^{s}=0$ are also assumed to be small. The change of functions along the hypersurface $x^{s}=0$ is characterized by the quantity $l$. Then $\varepsilon_{0}=\frac{2 h^{s}}{l} \ll 1$. The value $2 h^{s}$ is assumed to be finite positive, $2 h^{s}=$ finite $>0$. If the value $l$ is equal to the wavelength $\lambda$, i.e. $l=\lambda$, then in the hyperbolic operator this corresponds to the output to the characteristic defined by the principal part of the operator. 
Restriction on derivatives. We introduce a restriction on the class of differential operators in contrast to (Selezov, 2000) [9]. The derivative components parallel to the median surface of the hyperlayer must be even $u_{k, s}$ when $s=2,4, \ldots$, which narrows the class of problems under consideration. In the case of odd derivatives $u_{k, n}$, when $k=s$, the field can penetrate into the layer and the decomposition will not be suitable.

Expanding as a decrease in the dimension of the problem. Cauchy and Poisson, when considering the problem of elastodynamics in dimensional form for a layer, assumed that the thickness of the layer is small compared with the planar dimension. In the generalization to Euclidean space, this condition was noted above as the change in the unknown functions along the hypersurface is much larger than their change along the normal. The expansion of field functions in power series in terms of the coordinate relative to the median hypersurface leads to a degenerate problem of determining the coefficients of the series, which now depend only on the coordinates

$$
u_{i}\left(t, x^{2}, \ldots, x^{n-1}, x^{n}\right)=\sum_{k=1}^{\infty} u_{i k}\left(t, x^{2}, \ldots, x^{s-1}, x^{s}, \ldots, x^{n}\right)\left(x^{s}\right)^{k} .
$$

Thus, the dimension of the problem is reduced by 1 .

In this case, the expansions are valid for any field characterized by smooth (infinitely differentiable functions), so that there exist convergent expansions. In the formal construction of solutions, one can construct expansions in power series along the normal to the coordinate line, assuming the desired functions in the class $C^{\infty}$.

\section{Hyperbolic degeneration and the finiteness of the velocity of disturbances propaga- tion}

We consider a special case when the differential equations (1) and the boundary conditions (2) are represented as the sum of linear and nonlinear parts, where the linear operator $L$ is of higher order $p$ than the order $p_{1}$ of the nonlinear operator (Courant \& Hilbert, 1962) [11]

$$
\begin{aligned}
& a_{i l q}\left(x^{1}, \ldots, x^{n} ; \varepsilon_{1}, \ldots, \varepsilon_{\nu}\right) \frac{\partial^{q} u_{l}}{\partial x^{1\left(\alpha_{1}\right)} \ldots \partial x^{n\left(\alpha_{n}\right)}} \\
& +\hat{F}_{i}\left(x^{1}, \ldots, x^{n} ; u_{1}, \ldots, u_{k} ; \frac{\partial u_{l}}{\partial x^{1}}, \ldots, \frac{\partial u_{k}}{\partial x^{n}} ; \frac{\partial^{2} u_{l}}{\partial x^{1} \partial x^{2}}, \ldots ; \frac{\partial^{p 1} u_{l}}{\partial x^{1(p 1)}}, \ldots, \frac{\partial^{p 1} u_{k}}{\partial x^{n(p 1)}} ; \varepsilon_{1}, \ldots, \varepsilon_{\nu}\right)=P_{i} \quad \text { in } \quad \Omega \\
& i=\overline{1, k}, \quad l=\overline{1, k}, \quad q=\alpha_{1}+\alpha_{2}+\ldots+\alpha_{n}, \quad q=\overline{1, p}, \quad p_{1}=\overline{1,(p-1)}, \quad(3) \\
& \left\{b_{i l q}\left(x^{1}, \ldots, x^{n} ; \varepsilon_{1}, \ldots, \varepsilon_{\nu}\right) \frac{\partial^{q} u_{l}}{\partial x^{1\left(\alpha_{1}\right)} \ldots \partial x^{n\left(\alpha_{n}\right)}}\right. \\
& \left.+\hat{f}_{i}\left(x^{1}, \ldots, x^{n} ; u_{1}, \ldots ; \ldots, \frac{\partial^{p_{2}} u_{k}}{\partial x^{n\left(p_{2}\right)}} ; \varepsilon_{1}, \ldots, \varepsilon_{\nu}\right)\right\}\left.\right|_{x^{s}= \pm h^{s}}=Q_{j}^{ \pm} \quad \text { in } \quad \Omega \\
& j=p \cdot k, \quad q=\overline{1,(p-1)}, \quad p_{2}=\overline{1,(p-2)}, \quad l=\overline{1, k} . \quad(4)
\end{aligned}
$$

The system of equations (3), (4) when $\varepsilon_{r} \rightarrow 0$ will be called a degenerate system. If the hyperbolic system of differential equations (3) remains hyperbolic in parameter degeneracy, then such degeneration will be called a hyperbolic degeneracy.

It is assumed that the coefficients of the partial derivatives of the operator, which depend on the parameters $\varepsilon_{1}, \ldots, \varepsilon_{\nu}$, do not change the signs and do not vanish.

With degeneracy, equations of various types can be obtained. There are three possible cases: degeneracy to equations of hyperbolic, parabolic or mixed type. The question is - what is correct? 
According to the principle of hyperbolic degeneracy with respect to a small parameter, it is necessary that the original hyperbolic system degenerate also into a hyperbolic system. For this reason, only the limiting hyperbolic systems are of interest from the point of view of the finiteness of the velocity of propagation of perturbations and symmetry (Selezov, 2000) [9].

Differential equations and boundary conditions can be written in the form (Kythe,1996) [12]

$$
\begin{gathered}
L_{i} \equiv a_{i l q} \frac{\partial^{q} u_{l}}{\partial x^{1\left(\alpha_{1}\right)} \ldots \partial x^{n\left(\alpha_{n}\right)}}+F_{i}=P_{i} \quad \text { at } \quad \Omega, \quad t \geqslant 0,(i, l)=\overline{1, k}, q=\alpha_{1}+\alpha_{2}+\ldots+\alpha_{n}, q=\overline{1, p} \\
\left\{b_{j l q} \frac{\partial^{q} u_{l}}{\partial x^{1\left(\alpha_{1}\right)} \ldots \partial x^{n\left(\alpha_{n}\right)}}+f_{j}\right\}_{x^{s}= \pm h^{s}}=Q_{j}^{ \pm}, \quad j=p \cdot k, \quad q=\overline{1,(p-1)}, \quad l=\overline{1, k}, \quad \text { (6) }
\end{gathered}
$$

where (5) is a system of $k$ equations of the $p$ first order with $k$ unknown functions, which must be defined as its solutions satisfying the boundary conditions (6) and the initial conditions in the case of the initial-boundary value problem, so that the correct statement of the problem is guaranteed. The first term in (5) is the main part of the operator, the second term remains as part of the operator. In (6), the term $f_{j}$ is an operator of lower order than the first term. It is assumed that the coefficients $a_{i l q}$ and $b_{j l q}$ are constants, but they can depend on a small parameter $\varepsilon_{r} \ll 1$. Unlike equations of the general form (1), (2), the system of equations (5) can be classified by the type of partial differential equations. If the system of equations (5) is of hyperbolic type, then in the case of the well-posed Cauchy problem for (5), (6), solutions exist in the region in the form of weak propagating discontinuities (discontinuities of derivatives of the highest order in the differential operator). This corresponds to reality in actual physical media or systems, any perturbation propagates at a finite rate determined by the properties of the medium or system. The mathematical formulation of the finite-speed principle says that the solution of the Cauchy problem with completely defined initial data is finite with respect to spatial derivatives for every fixed value of the time coordinate (Kalashnikov, 1979) [13]. It should be noted that the main part of the operator is responsible for the solvability of the initial-boundary value problem for the Cauchy-Kovalevskaya (Misokhata, 1965) [14].

It is necessary to obtain hyperbolic approximations, i.e. to construct a mapping of the original space $\mathbb{R}^{n}\left(\varepsilon_{r}\right)$ into a degenerate space $\mathbb{R}^{n}\left(\varepsilon_{r}\right) \rightarrow \mathbb{R}^{n}$ that satisfies the condition of limiting correctness to be of hyperbolic type; the condition of finite velocity of perturbation propagation $[5,16]$. In this case, new functions $\hat{u}(x)$ appear in the degenerate space instead of functions $u(x)$.

In what follows we consider the case of coordinate degeneracy $\varepsilon_{0}=2 h / l=$ finite. The expansion of field functions in power series in a degenerate coordinate $s$ relative to the mid-hypersurface leads to a degenerate problem of determining the coefficients of series that now depend only on the $n-1$ coordinates

$$
u_{i}\left(t, x^{2}, \ldots, x^{n-1}, x^{n}\right)=\sum_{k=1}^{\infty} u_{i k}\left(t, x^{2}, \ldots, x^{s-1}, x^{s}, \ldots, x^{n}\right)\left(x^{s}\right)^{k} .
$$

The substitution of (7) into partial differential equations (5) and boundary conditions (6) yields recurrence relations from equations (5) and the set of systems of differential equations of infinite order from (6) in. Recurrence relations allow us to express all the coefficients in increasing approximations in terms of several initial ones.

The next step is the truncation of these infinite systems, which is possible in different ways, while preserving the terms corresponding to different rules. One can express all of the recurrence relations in terms $u_{i k}$ of a minimal finite number of unknown functions corresponding to the number of systems of differential equations. Substitution of these functions into truncated equations leads to resolving equations, which makes it possible to obtain various approximations, i.e. simplified models. The rules for preserving the terms must be of the form that this system is of the hyperbolic type.

The necessary condition. A necessary condition for the existence of a finite velocity of perturbation propagation in the $n$-th approximation is the hyperbolicity of the operator of the $n$-th order. 
To construct the hyperbolic approximation of the $n$-th order, it is necessary to keep all spatiotemporal differential operators up to a given order $n$ in infinite systems. The preservation of all the terms of the operator up to a certain order gives the main part of the operator of hyperbolic type.

The sufficient condition for the existence of the finiteness of the velocity of perturbation propagation is the correct formulation of the IBV problem (Misokhata, 1965) [14].

For example, the preservation of operators up to the order 4 in an $n$-dimensional Euclidean space leads to a hyperbolic approximation corresponding to the Timoshenko equation, but not containing a correction factor of the thickness-shear type. The keeping of operators up to the 6 -th order gives a new higher approximation - a generalized hyperbolic equation.

\section{Generalized hyperbolic equation of propagation of bending waves in elastic layer}

Following the foregoing, let us consider the construction of degenerate models for the case $\mathbb{R}^{4}$. This is the problem of elastodynamics for a layer. The mathematical formulation of the corresponding IBV-problem for an elastic isotropic medium in terms of displacements $u_{1}, u_{2}, u_{3}$ that depend on the orthogonal coordinates $x_{1}, x_{2}, x_{3}$ and the time $t$ is represented as follows: find the vector function $\boldsymbol{u}$ $\left(x_{1}, x_{2}, x_{3}, t\right)$ as a solution of the equations in $\Omega \times[0, T], T>0$

$$
\nabla^{2} u_{k}+(1+\lambda / G) \partial_{k}(\boldsymbol{\nabla} \cdot \boldsymbol{u})+P_{k}=\partial_{t t} u_{k}, \quad k=1,2,3,
$$

satisfying the boundary conditions

$$
\begin{gathered}
\left.\sigma_{33}\right|_{x_{3}=\xi / 2}=q^{+}\left(x_{1}, x_{2}, t\right),\left.\quad \sigma_{33}\right|_{x_{3}=-\xi / 2}=q^{-}\left(x_{1}, x_{2}, t\right) \\
\left.\sigma_{3 i}\right|_{x_{3}=\xi / 2}=p_{i}^{+}\left(x_{1}, x_{2}, t\right),\left.\quad \sigma_{3 i}\right|_{x_{3}=-\xi / 2}=p_{i}^{-}\left(x_{1}, x_{2}, t\right), \quad(i=1,2)
\end{gathered}
$$

and the initial conditions

$$
\left.u_{k}\right|_{t=0}=0,\left.\quad \partial_{t} u_{k}\right|_{t=0}=0, \quad k=1,2,3 .
$$

The components of the displacement vector are represented in the form of power series in $x_{3}$

$$
u_{i}\left(x_{1}, x_{2}, x_{3}, t\right)=\sum_{\nu=0}^{\infty} u_{i \nu}\left(x_{1}, x_{2}, t\right) x_{3}^{\nu}, \quad i=1,2,3 .
$$

The functions $u_{i \nu}$ are assumed to be differentiable as many times as required, all the derivatives $u_{i \nu}$ are continuous, and the series (11) converge uniformly.

Mass forces $P_{k}$ are not taken into account in the future. The values are equal $\xi=2 h, \nabla \equiv$ $\frac{\partial}{\partial x_{1}} \boldsymbol{e}_{1}+\frac{\partial}{\partial x_{2}} \boldsymbol{e}_{2}+\frac{\partial}{\partial x_{3}} \boldsymbol{e}_{3}$ is the gradient operator, $\boldsymbol{e}_{k}, k=1,2,3$ is the basis vector; $\{\cdot\}$ is the symbol of scalar multiplication. The symbols $\partial_{k}$ and $\partial_{t}$ are the partial derivatives with respect to the coordinate $x_{k}$ and time $t, \nabla^{2}=\boldsymbol{\nabla} \cdot \boldsymbol{\nabla}$ is the Laplacian. The expression for the components of the stress tensor has the form

$$
\sigma_{i k}=\lambda u_{n, n} \delta_{i k}+G\left(u_{i, k}+u_{k, i}\right) .
$$

Here $\delta_{i k}=\left\{\begin{array}{c}0, \quad i \neq k, \\ 1, \quad i=k\end{array}\right.$ is the Kronecker symbol, $G$ and $\lambda$ are the Lame constants, expressed in terms of the Young's modulus $E$ and Poisson's ratio $\nu, G=E / 2(1+\nu), \lambda=E \nu /(1+\nu)(1-2 \nu)$. The agreement on summation over repeated indices is meant, and indices after the comma denote the partial differentiation with respect to the corresponding coordinate.

Dimensionless quantities are introduced using the formulas, taking as the characteristic thickness $2 h(\mathrm{~m})$, shear wave velocity $c_{s}(\mathrm{~m} / \mathrm{s})$, density $\rho(\mathrm{kg} / \mathrm{m})$

$$
u_{k}^{*}=\frac{1}{2 h} u_{k}, \quad\left(x_{1}^{*}, x_{2}^{*}\right)=\frac{1}{2 h}\left(x_{1}, x_{2}\right), \quad t^{*}=\frac{c_{s}}{2 h} t, \quad q^{*}=\frac{1}{G} q, \quad h^{*}=\frac{1}{2}, \quad c_{s}^{2}=G / \rho .
$$

Mathematical Modeling and Computing, Vol.5, No. 1, pp. 88-97 (2018) 
When investigating the propagation of waves, dimensionless variables are introduced $l^{*}=\frac{1}{2 h} l$ is the wavelength, $c^{*}=\frac{c}{c_{s}}$ is the phase velocity. The generalized differential equation with respect to the transverse coordinate $u_{3}=w_{0}$ has the form (the asterisk is omitted)

$$
\begin{gathered}
\left\{\left[\left(\frac{\partial^{2}}{\partial t^{2}}+a_{1} \nabla^{2} \nabla^{2}\right)_{\mathrm{K}} a_{2} \frac{\partial^{2}}{\partial t^{2}} \nabla^{2}+a_{3} \frac{\partial^{4}}{\partial t^{4}}\right]_{\mathrm{TM}}-b_{1} \nabla^{2} \nabla^{2} \nabla^{2}+b_{2} \frac{\partial^{2}}{\partial t^{2}} \nabla^{2} \nabla^{2}-b_{3} \frac{\partial^{4}}{\partial t^{4}} \nabla^{2}+b_{4} \frac{\partial^{6}}{\partial t^{6}}\right\}_{\mathrm{TMC}} \quad w_{0} \\
=\left\{\left[1-d_{1} \nabla^{2}+d_{2} \frac{\partial^{2}}{\partial t^{2}}\right]_{\mathrm{TM}}+d_{3} \nabla^{2} \nabla^{2}-d_{4} \frac{\partial^{2}}{\partial t^{2}} \nabla^{2}+d_{5} \frac{\partial^{4}}{\partial t^{4}}\right\}_{\mathrm{TMC}}\left(q^{+}-q^{-}\right) . \quad(12)
\end{gathered}
$$

In (12) the following notations are accepted: $w_{0}\left(x_{1}, t\right)$ is the transverse displacement (deflection), $t$ is the time, $\left(q_{1}-q_{2}\right)$ is the lateral load, coefficients are equal to

$$
\begin{gathered}
a_{1}=\frac{1}{6(1-\nu)}, \quad a_{2}=\frac{2-\nu}{6(1-\nu)}, \quad a_{3}=\frac{7-8 \nu}{48(1-\nu)}, \quad b_{1}=\frac{1}{120(1-\nu)}, \quad b_{2}=\frac{4 \nu^{2}-16 \nu+1}{480(1-\nu)^{2}}, \\
b_{3}=\frac{16 \nu^{2}-37 \nu+19}{5760(1-\nu)^{2}}, \quad b_{4}=\frac{64 \nu^{2}-104 \nu+41}{7680(1-\nu)^{2}}, \quad d_{1}=\frac{2-\nu}{8(1-\nu)}, \quad d_{2}=\frac{1}{8}, \\
d_{3}=\frac{\nu^{2}-4 \nu+3}{384(1-\nu)^{2}}, \quad d_{4}=\frac{4 \nu^{2}-12 \nu+7}{768(1-\nu)^{2}}, \quad d_{5}=\frac{1}{384} .
\end{gathered}
$$

The operator with index K corresponds to the Bernoulli-Euler equation (extended to plates by Kirchhoff). The operator with the TM index corresponds to the Timoshenko equation (extended to the plates by Ufland and developed by Mindlin). The Rayleigh equation enters into the operator TM at $a_{3}=0$. The operator with the TMC index corresponds to the generalized equation (constructed by Selezov). From the above analytical construction follows as a special case the Timoshenko equation, but without introducing a correction parameter - the coefficient of shear.

\section{Analysis of the solvability of Cauchy-Poisson approximations}

After introducing the dimensionless quantities presented above, as well as carrying out cumbersome and long calculations, the coefficients $\alpha_{i k}, b_{m n}$ were found, which, when introducing dimensionless quantities dependent only on the Poisson ratio $\nu$. They were subsequently calculated with a Poisson's ratio $\nu=0.3$ characterizing many materials. Their values are equal to $a_{1}=0.238, a_{2}=0.405$, $a_{3}=0.137, b_{1}=0.012, b_{2}=0.028, b_{3}=0.003, b_{4}=0.004$.

\begin{tabular}{|c|c|c|c|}
\hline \multicolumn{2}{|c|}{6} & \multicolumn{2}{|c|}{4} \\
\hline 0 & 0.661 & 0 & 0.900 \\
1 & 0.701 & 1 & 0.847 \\
2 & 0.709 & 2 & 0.744 \\
3 & 0.646 & 3 & 0.643 \\
4 & 0.566 & 4 & 0.557 \\
5 & 0.495 & 5 & 0.487 \\
6 & 0.436 & 6 & 0.430 \\
7 & 0.388 & 7 & 0.383 \\
8 & 0.348 & 8 & 0.344 \\
9 & 0.315 & 9 & 0.312 \\
10 & 0.287 & 10 & 0.285 \\
\hline
\end{tabular}

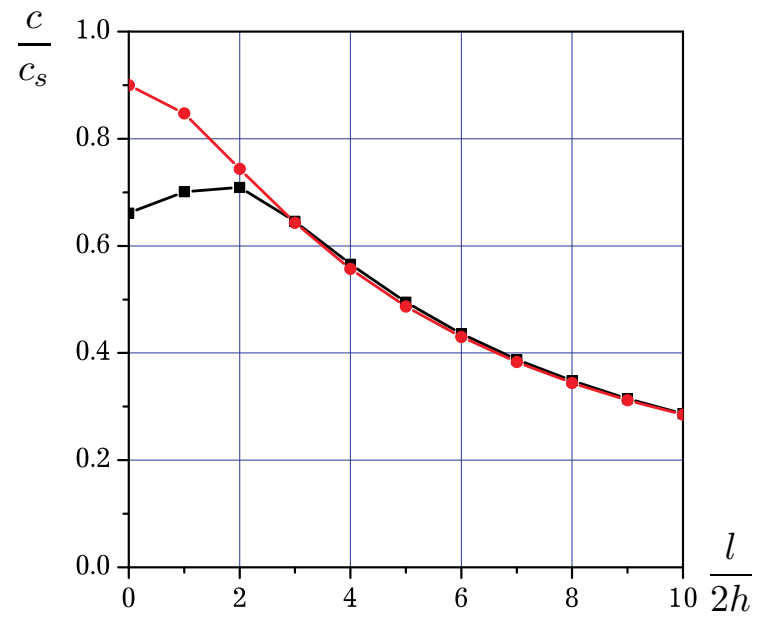

Fig. 1. The solvability of hyperbolic approximations of the 6-th and 4-th orders of the propagation of bending waves in a beam strip. 
The conditions for the solvability of hyperbolic approximations of the 6-th and 4-th orders are obtained for the propagation of transverse (bending) waves in a beam strip. The results of the computer calculations are shown in Fig. 1.

The solvability of the approximations was investigated by substituting in equation (12) a solution of the traveling wave type $\exp [i(k x-\omega t)]$, where $k=2 \pi / l, \omega=c 2 \pi / l$.

It is shown that the 6 -th order approximation has one real root in the wavelength interval $l \in[0,10)$ (Fig. 1), and for $l>10$ it has one real and two complex-conjugated roots. The above construction of hyperbolic approximations made it possible to obtain analytical expressions for the coefficients of the expansions, in contrast to the indefinite correcting coefficient characterizing the thickness shear entering in the Timoshenko equation.

\section{The model of Timoshenko. A new insight}

A new look at the refined equations of the Timoshenko type for rods, plates and shells is presented in accordance with the fundamental studies of Maxwell, Einstein, and Landau on the propagation of perturbations with finite velocity. J. Maxwell after fundamental research in the field of electromagnetism (Maxwell, 1864) [1] first introduced the hyperbolic equation of propagation of waves with finite velocity in gases (Maxwell, 1867) [2] instead of the parabolic equation describing the perturbation propagation with infinite velocity.

S. P. Timoshenko (1921) [15] first generalized the parabolic equation for the propagation of flexural vibrations of a beam to a hyperbolic equation (1921), applying the phenomenological approach, so that the normal does not remain normal to the middle surface under bending deformations of the beam, which is not taken into account by the model continuous medium. In the classical models of Bernoulli-Euler, Kirchhoff (1850) [16], Rayleigh (1889) [17], the normal remains normal. The effects of Timoshenko appear locally in the presence of sharp inhomogeneities, in wave theory these are short waves. In this case, it is necessary to apply a more general theory than the classical one, for example, the theory of Cosserat (1909).

It is impossible to change the type of the differential operator by any correction factor; it is possible only by selecting this coefficient to approximate the description to the model predicted by the continuous medium.

Timoshenko's generalization is analogous to that which occurs in the construction of other hyperbolic models, when higher order effects are taken into account that go beyond the framework of classical traditional theories. For example, the heat propagation model (Cattaneo, 1948) [18], in this case, the difference from the classical theory was later explained by nonequilibrium thermodynamics (Lykov, 1967) [19], the diffusion model (Davydov, 1935) [21] takes into account the collision of particles in contrast to the classical theory. We also note the model of hyperbolic turbulence (Monin 1955) [20], the equation of Smoluchowski (Davies, 1954) [22], the equation of statistical processes (Fock, 1926) [23], the sedimentation equation (Selezov, 2014) [24].

Application of the Cosserat theory. In the classical theory of continuous media, it is assumed that the forces acting collinearly act on an infinitesimal element (Fig. 2).
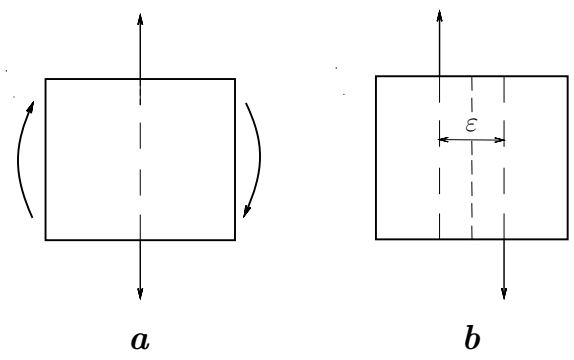

Fig. 2. Effects of forces on an infinitesimal element.
In the presence of a moment field, this field (Fig. 2a), can be regarded as appearing due to the noncollinear forces (Fig. 2b). Cosserat (1909) [18] assumed the collinearity of forces and introduced the moment $\theta$. However, in any case (Fig. 2), this can be understood as a discontinuity. This is in accordance with Timoshenko's equation, when the normal to the middle surface does not remain normal after deformation. Thus, the introduction of moment stresses can be understood as the action 
of noncentral forces, and this is a violation of continuity, and when the beam is bent, the normal does not remain a normal to the deformed middle surface. This situation is essentially postulated in the derivation of the Tymoshenko equation: the slope of the tangent to the bending curve $\partial w / \partial x=\psi+\gamma$ is represented in the form where $\psi$ the bending deformation, $\gamma$ shear deformation. At high frequencies or sharp inhomogeneities this will manifest itself.

We give the equations of the Cosserat theory. Without loss of generality, we can obtain a plane problem from (8)-(11) by rotating about a vertical axis $O x_{3}$. In accordance with the dimensionless quantities introduced above and supplemented by the Cosserat parameters (the asterisks are further omitted)

$$
\alpha^{*}=\frac{\alpha}{(2 h)^{2}}, \quad \beta^{*}=\frac{\beta}{(2 h)^{2}}, \quad \gamma^{*}=\frac{\gamma}{(2 h)^{2}}, \quad \varepsilon_{k}^{*}=\frac{\varepsilon_{k}}{(2 h)^{2}}, \quad j^{*}=\frac{c_{s}^{2}}{(2 h)^{2}} j .
$$

The equations for the displacement vector $\boldsymbol{u}$ and the rotation vector $\boldsymbol{\theta}$ have the form

$$
\begin{gathered}
V \nabla^{2} \boldsymbol{u}+(1+\lambda / G) \boldsymbol{\nabla}(\boldsymbol{\nabla} \cdot \boldsymbol{u})-\alpha \boldsymbol{\nabla} \times \boldsymbol{\nabla} \times \boldsymbol{u}+2 \alpha \boldsymbol{\nabla} \times \boldsymbol{\theta}=\partial_{t t} \boldsymbol{u}, \\
(\beta+2 \gamma) \boldsymbol{\nabla}(\boldsymbol{\nabla} \cdot \boldsymbol{\theta})-\left(\gamma+\varepsilon_{k}\right) \boldsymbol{\nabla} \times \boldsymbol{\nabla} \times \boldsymbol{\theta}+2 \alpha \boldsymbol{\nabla} \times \boldsymbol{u}-4 \alpha \boldsymbol{\theta}=j \partial_{t t} \ddot{\boldsymbol{\theta}} .
\end{gathered}
$$

Timoshenko's generalization. From the point of view of the theory of differential operators, Timoshenko's generalization is essentially nontrivial, since in this case a parabolic operator of a higher order (the fourth, not the second) is generalized, in contrast to all previous generalizations (diffusion, heat, etc.).

For clarity, we give the equations describing the propagation of one-dimensional waves, which follow from (12) under rotation with respect to the vertical axis normal to the middle surface. The Bernoulli-Euler equation (1695, 1744), extended to plates by Kirchhoff (1850) [16]

$$
\frac{\partial^{2} w}{\partial t^{2}}+\frac{D}{\rho h} \frac{\partial^{4} w}{\partial x^{4}}=0
$$

The Rayleigh equation taking into account the inertia of rotation (Rayleigh, 1889) [17]

$$
\frac{\partial^{2} w}{\partial t^{2}}+\frac{D}{\rho h} \frac{\partial^{4} w}{\partial x^{4}}-\left(\frac{I}{h}\right) \frac{\partial^{4} w}{\partial t^{2} \partial x^{2}}=0
$$

The Timoshenko equation taking into account the shift (Timoshenko, 1921) [15]

$$
\frac{\partial^{2} w}{\partial t^{2}}+\frac{D}{\rho h} \frac{\partial^{4} w}{\partial x^{4}}-\left(\frac{D}{k_{s}^{2} G h}+\frac{I}{h}\right) \frac{\partial^{4} w}{\partial t^{2} \partial x^{2}}+\frac{\rho I}{k_{s}^{2} G h} \frac{\partial^{4} w}{\partial t^{4}}=0 .
$$

Equation (15), including (13) and (14), was presented in a substantial article by S. P. Timoshenko in 1921 (Timoshenko, 1921) [15] and was widly distributed as Timoshenko's equation.

\section{Conclusion}

A generalization of the Cauchy-Poisson method for the $n$-dimensional Euclidean space with restrictions on differential operators and a small number of degeneracy parameters has been presented. Conditions for the degeneracy of hyperbolic approximations and the finiteness of the propagation velocity of perturbations are given. A generalized equation for the propagation of bending waves in a layer has been obtained as a particular case when $n=4$. The Timoshenko equation has been considered as a non-trivial generalization of a parabolic operator of a higher order than in the previous study. From our research that generalizes known parabolic models to hyperbolic it follows that the construction of higher hyperbolic approximations does not improve the accuracy of describing physical phenomena. 
[1] Maxwell J. C. A dynamical theory of the electromagnetic field. Cambridge University Press (1864).

[2] Maxwell J. C. On the dynamical theory of gases. Phil. Trans. Roy. Soc. 157, 49-88 (1867).

[3] Einstein A. The meaning of relativity. Princeton University Press (1950).

[4] Weber J. General relativity and gravitational waves. New York, Interscience Publishers (1961).

[5] Selezov I. T., Kryvonos Yu. G. Wave hyperbolic models propagation of perturbations. Kiev, Naukova Dumka (2015).

[6] Selezov I. T., Kryvonos Yu. G. Modeling medicine propagation in tissue: generalized statement. Cybernetics and Systems Analysis. 53 (4), 535-542 (2017).

[7] Cauchy A. L. Sur l'équilibre et le mouvement d'une lame solide. Exercices Math. 3, 245-326 (1828).

[8] Poisson S.D. Mémoire sur l'équilibre et le mouvement des corps élastiques. Mém. Acad. Roy. Sci. 8, $357-570$ (1829).

[9] Selezov I. T. Degenerated hyperbolic approximation of the wave theory of elastic plates. Ser. Operator Theory. Advances and Applications. Differential Operators and Related Topics. Proc. of Mark Krein Int. Conf., Ukraine, Odessa, 18-22 August 1997. Basel/Switzerland, Birkhauser. Vol. 117, 339-354 (2000).

[10] Dunford N., Schwartz J. T. Linear operators. Part II. Spectral theory. Self adjoint operators in Hilbert space. New York, London, Interscience Publishers (1963).

[11] Courant R., Hilbert D. Methods of mathematical physics. Vol.1, 2. Interscience, New York-London (1962).

[12] Kythe P. K. Fundamental solutions for differential operators and applications. Birkhauser Boston (1996).

[13] Kalashnikov A.S. The concept of a finite rate of propagation of a perturbation. Russian Math. Surveys. 34 (2), 235-236 (1979).

[14] Misokhata C. The theory of partial differential equations. University Kioto (1965).

[15] TimoshenkoS.P. On the correction for shear of the differential equation for transverse vibrations of prismatic bar. Philosophical Magazine and Journal of Science. 41 (245), 744-746 (1921).

[16] Kirchhoff G. Über das Gleichgewicht und die Bewegung einer elastischen Scheibe. Journal für die reine und angewandte Mathematik. 40 (1), 51-58 (1850).

[17] Rayleigh D. On the free vibrations of an infinite plate of homogeneous isotropic elastic matter. Proc. London Math. Soc. 10, 225-237 (1889).

[18] CosseratE.\& F. Théorie de Corps déformables. Hermann, Paris (1909).

[19] Cattaneo C. Sulla conduzione del calore. Atti Semin. Mat. Fis. della Università di Modena. 3, 3-21 (1948).

[20] Luikov A. V. Application of irreversible thermodynamics methods to investigation of heat and mass transfer. Int. J. Heat Mass Transfer. 9 (2), 139-152 (1966).

[21] Davydov B. I. The diffusion equation with allowance for the molecular velocity. Reports of the Academy of Sciences of the USSR. 2 (7), 474-475 (1935).

[22] Monin A. M. On diffusion with finite velocity. Izv. Academy of Sciences of the USSR, ser. geogr. 3, 234-248 (1955).

[23] Davies R. W. The connection between the Smoluchowski Equation and the Kramers-Chandrasekhar equation. Phys. Rev. 93 (6), 1169-1171 (1954).

[24] Fock V. A. Solution of a problem in the theory of diffusion by the method of finite differences and its application to diffusion of light. Proceedings of the State Optical Institute. Vol. 4, Issue 34, 1-32. §13 (1926). Connection with differential equations and an expression for diffusion. 29-31.

[25] Selezov I. Extended models of sedimentation in coastal zone. Vibrations in Physical Systems. 26, 243-250 (2014). 


\title{
Узагальнення та застосування метода Коші-Пуассона до еластодинаміки шару та рівняння Тимошенко
}

\author{
Селезов I. \\ Інститут гідромеханіки НАН Украӥни, \\ вул. Желябова, 8/4, 03057, Київ, Украӥна
}

Метод Коші-Пуассона узагальнено на $n$-вимірний евклідів простір так, щоб отримати диференціальні рівняння в часткових похідних вищого порядку. Наведено застосування до побудови гіперболічних апроксимацій, що узагальнюють та доповнюють попередні дослідження. В евклідовому просторі вводять обмеження на похідні. Розглянуто гіперболічне виродження за параметрами та його реалізація у вигляді необхідних і достатніх умов. Як окремий випадок 4-вимірного евклідового простору, зберігаючи оператори до 6-го порядку, отримано узагальнене гіперболічне рівняння поперечних (згинних) коливань пластин з коефіцієнтами, залежними тільки від числа Пуассона. Це рівняння містить як окремі випадки всі відомі рівняння Бернулі-Ейлера, Kipхгофа, Релея, Тимошенко. Зазначено, що уточнене рівняння згинних коливань балки, вперше представлене Тимошенко, потрібно розглядати як розвиток досліджень Максвелла і Ейнштейна про поширення збурень зі скінченою швидкістю в середовищі. Вперше відзначено відповідність з теорією Коссера.

Ключові слова: метод Коші-Пуассона, евклідів простір, диференціалъне рівняння в часткових похідних, еластодинаміка, шар, гіперболічні апроксимащї, рівняння Тимошенко.

2000 MSC: 74H10, 74J05, 74K20

УдК: 531.4 